\title{
Cryo Soft X-ray Tomography and Other Techniques at Diamond Light Source
}

\author{
M.C. Darrow ${ }^{1}$, M. Harkiolaki ${ }^{1}$, M. Spink ${ }^{1}$, I. Luengo ${ }^{1,2}$, M. Basham ${ }^{1}$ and E.M.H. Duke ${ }^{1}$
}

1: Diamond Light Source, Harwell Science and Innovation Campus, Chilton, Didcot. OX11 0DE. UK 2: The University of Nottingham, Computer Vision Laboratory, Nottingham. NG7 2RD. UK

Diamond Light Source is the UK's national synchrotron facility, providing an intense source of X-ray, ultra violet and infra-red radiation to both the academic and industrial scientific community. Recently, a microscope dedicated to cryo soft X-ray tomography (cryoSXT) of biological samples has been commissioned. This microscope will allow data to be collected from cells and other similar samples vitrified on standard 3mm diameter electron microscopy grids. The X-ray microscope was purchased as part of a collaboration between Xradia (now Zeiss) and Diamond Light Source and delivered along with an off-line source of X-rays (nitrogen plasma source) in 2012. Since then the system has undergone extensive commissioning, including upgrading the light microscope used for sample selection to both increase the magnification and to incorporate the ability to measure fluorescence from suitably labelled samples. The microscope is now located on the beamline and using X-rays from one of the storage ring bending magnets for illumination rather than the plasma source. Currently, the beamline is operating a fixed energy $(500 \mathrm{eV})$ within the so-called "water window", so image formation is via the naturally occurring absorption differential between carbon and oxygen [1,2]. This allows for the imaging of whole, fully-hydrated cells, without sectioning or staining techniques, at approximately $40 \mathrm{~nm}$ resolution.

Huntington's Disease (HD), a neurodegenerative disorder characterized by movement and executive function disruption, is linked to an expanded and unstable CAG trinucleotide repeat which translates as a polyglutamine $(\mathrm{Q})$ repeat in the protein product. Healthy controls demonstrate a fairly broad range of trinucleotide repeats and 40+ repeats have been described as pathological. Due to this expansion, exon 1 of the Huntingtin protein (HTT) is aberrantly processed by cellular machinery leading to an unintended cleavage product (mHTT). Aggregates of mHTT exon 1 have been found in mouse models and in patient brain [3].

PC-12 cells were used as an HD model to study cell morphology changes due to the presence of mHTT. Cells expressing mHTT-GFP exon 1 [4] were grown on gold finder grids, cryo-immobilised by plunge freezing, and imaged*. GFP fluorescence was used to identify cells of interest prior to imaging in cryoSXT. Tilt series were collected and reconstructed (Figure 1), and segmentation was carried out using a new software tool called SuRVoS (Super-Region Volume Segmentation) Workbench [5, 6].

Super-Region Volume Segmentation (SuRVoS) Workbench combines the human expert's knowledge with data representation, machine learning and active learning techniques. This workbench is designed to semi-automatically segment large volumes of data with the input of a user and to guide the user through the process. SuRVoS uses hierarchical layers of super-regions to partition volume data into more meaningful regions. The super-regions are then used to segment a portion of the data and with this as input, a computational classifier is trained and applied to the whole volume in real time. The user then accepts voxels that are classified with high certainty and can repeat this model training step to further refine the results if necessary. This strategy reduces manual segmentation, greatly decreasing the amount of user time spent on segmenting, and removes manual delineation of boundaries, potentially reducing subjectivity. The resulting segmentations can be measured and classified within the tool and exported for 
further visualization.

SuRVoS was used to annotate cellular features based on their inherent properties. Cytoplasmic organelles were classified using specific characteristics of the data, such as the average intensity of the organelle, the size of the organelle, the variance of the voxels that make up the organelle and the location of the organelle in the 3D volume. Next, population statistics for each organelle class, such as the average and standard deviation of the class's size, shape, intensity and variance were used to describe the morphology of a nonperturbed cell, allowing the identification of changes due to perturbations, in this case, the presence of mHTT aggregates.

As a whole, cryoSXT is a powerful technique that can describe the effects of various cellular perturbations such as disease, infection or treatment, in a whole cell context.

[1]: R Carzaniga, M-C Domart, LM Collinson and E Duke, Protoplasma 251 (2014), p. 449-58.

[2]: MA Le Gros, G McDermott and CA Larabell, Curr Opin Struct Biol 15 (2005), p. 593-600.

[3]: DM Hatters, Curr Top Med Chem, 12 (2012) p. 2611-22.

[4]: BL Apostol, et al., Proc Natl Acad Sci USA 100 (2003), p. 5950-5.

[5]: I Luengo, AP French and M Basham, (2017). DiamondLightSource/SuRVoS: Version 1.0. https://doi.org/10.5281/zenodo. 247547

[6]: I Luengo, et al., In Review, (2017) SuRVoS: Super-Region Volume Segmentation Workbench.

*: Samples courtesy of Wei Dai, Rutgers University, NJ, USA and Patrick Mitchell, Baylor College of Medicine, TX, USA

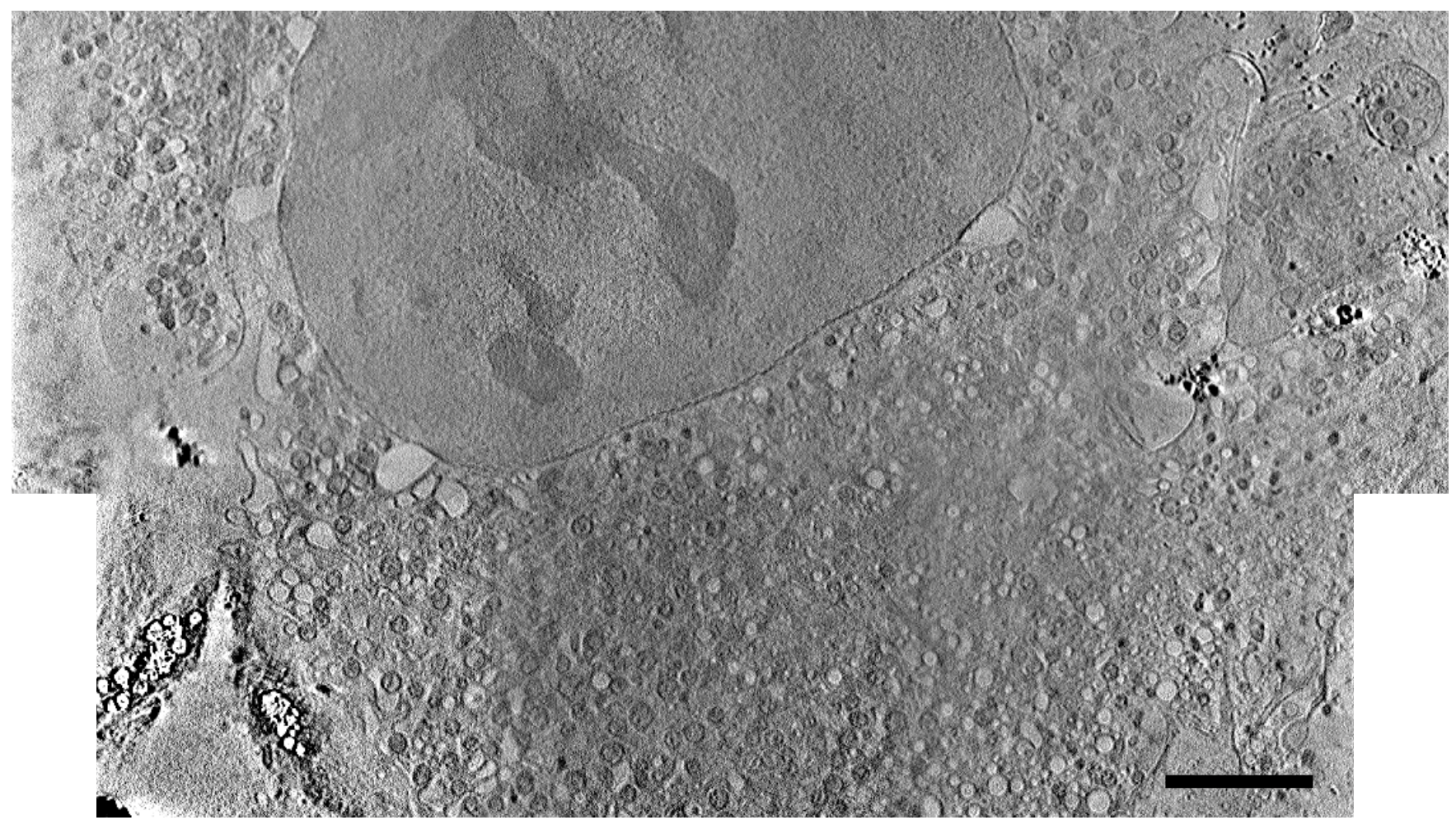

Figure 1. Seven overlapping tilt series of a mHTT expressing cell were collected using cryoSXT, reconstructed and merged in $3 \mathrm{D}$ providing the cellular context of the disease state. Scale bar is $5 \mu \mathrm{m}$. 